\title{
El difícil camino del control sanitario de la tuberculosis
}

\author{
CARLOS PEÑA M.**** y VICTORINO FARGA C.**
}

\section{The difficult road to achieve sanitary tuberculosis control}

Tuberculosis continues to be a worldwide prevalent disease. Chile has established a tuberculosis control program since the earliest 70 s which contributed to decrease the annual incidence of tuberculosis by 50\% every decade, reaching the rate of 20 per 100.000 inhabitants the year 2000 ("early phase of tuberculosis elimination as a public health problem"). Unfortunately since this time the descent rate is becoming slower and between years 2009-2011 has achieved a stationary rate of $13 \times 100.000$. This deterioration in the epidemiological situation of tuberculosis in Chile runs in parallel with the successive reductions in the budget assigned for its control. A higher incidence of tuberculosis in high risk groups (HIV population, migrants, prisoners and elderly people) were not responsible for this epidemiologic deterioration. Lack of diagnosis in smear positive pulmonary tuberculosis patients is the most important source of persistent community infection. Only $25 \%$ of the estimated goal of sputum smear screening among medical consultations in primary care was achieved. Between the years 20062010193.416 less sputum smear examinations were done. This means that nearly 966 smear positive tuberculosis patients were no identified as we know that about $0.5 \%$ smears are positive in this setting. A shortage of funds for tuberculosis in our country could increase its incidence as it was shown in Brazil, Cuba and Uruguay and would demand larger resources to strengthen the tuberculosis program. This epidemiological scene is worse in the Metropolitan Region where $40 \%$ of the country population concentrated $60 \%$ of the incidence of tuberculosis the year 2011. In this area we found counties with the highest rates. There is the need to strengthen the tuberculosis program in these areas and to organize teams in the primary care settings in order to increase case finding through the performance of sputum smears in symptomatic adult patients.

Key words: Tuberculosis control, Tuberculosis epidemiology, case finding strategy.

\section{Resumen}

La tuberculosis (TB) sigue siendo una patología prevalente a nivel mundial. Chile tiene un Programa de Control de la Tuberculosis eficaz desde el año 1973, el que ha permitido disminuir la incidencia anual de la enfermedad en 50\% cada década, hasta alcanzar el año 2000 una tasa de $20 \times 100.000$ habitantes ("umbral de la etapa de eliminación"). Sin embargo, desde entonces el país ha presentado un descenso menor, llegando a una situación estacionaria, con tasas cercanas a $13 \times 100.000$ los años 2009-2011. Este deterioro en la situación epidemiológica de la tuberculosis en Chile corre paralelo a las sucesivas reducciones del presupuesto asignado a su control. La mayor incidencia de tuberculosis en pacientes VIH, población migrante, población privada de libertad y adultos mayores han demostrado no ser los responsables de este retroceso epidemiológico. La mayor causa de este deterioro se debe a la pérdida de la capacidad diagnóstica de los casos transmisores de la enfermedad en la comunidad (casos pulmonares baciliferos), reflejado por el bajo índice de pesquisa (número de baciloscopias realizadas por cada 1.000 consultas de morbilidad general de adultos en la atención primaria), que es 12,8 $x 1.000$ a nivel nacional, en circunstancias que la meta es $50 \times 1,000$. Esto ha determinado la reducción de 193.416 baciloscopias de pesquisa entre los años 2006-2010, lo que sin duda mantiene en circulación un número estimado de 966 pacientes contagiosos no diagnosticados en este período de 4 años.

* Equipo Técnico de Tuberculosis. Servicio de Salud Metropolitano Central.

** Asesor del Programa de Control de la Tuberculosis, Ministerio de Salud de Chile. 
Esto hace temer que en Chile se pudiera producir próximamente un nuevo aumento de la tuberculosis, es decir, una reversión epidemiológica, como la que se observó en Brasil, Cuba y Uruguay cuando se redujeron los recursos destinados a sus programas antituberculosos. El control de este rebrote, en esos países, requirió de la inyección de ingentes recursos económicos. Este deterioro epidemiológico es más intenso en la Región Metropolitana, que en 2011 concentraba en el 40\% de la población nacional el 63\% de los casos de tuberculosis. Las tasas en esta población han permanecido sin cambios durante los últimos 6 años. Además, la Región Metropolitana presenta zonas de muy alta incidencia, como son las comunas de Santiago (tasa 71,4 x 100.000), Estación Central (51,9 x 100.000), Independencia (46,8 $x$ 100.000), San Joaquín (41,4 x 100.000) y Recoleta (35,3 x 100.000). Estas zonas pasan a ser prioritarias y requerirán de una intervención sanitaria más intensa y del reforzamiento de los equipos de tuberculosis en la atención primaria para que a través de la colaboración de todo su personal de salud aumenten la pesquisa bacteriológica de la tuberculosis pulmonar bacilifera.

Palabras clave: Control de la tuberculosis, epidemiología de la tuberculosis, estrategia en detección de casos.

\section{Situación mundial de la tuberculosis}

La tuberculosis ha sido catalogada por la Organización Mundial de la Salud (OMS) como una Emergencia Mundial. En 2010, la OMS estimó la existencia de 8,8 millones de casos de tuberculosis a nivel global y una incidencia de 128 casos nuevos por 100.000 habitantes ${ }^{1}$. La distribución geográfica de esta enfermedad muestra que las zonas de mayor endemia son el África subsahariana y el Sudeste Asiático, especialmente la India. Ese año, del total de casos de tuberculosis notificados en el mundo, India y China sumaron el $40 \%$ y África el $24 \%{ }^{2}$. En el África sub-sahariana la tasa alcanzó el año 2011 una incidencia de $260 \times 100.000^{3}$. Desafortunadamente, los países con mayor prevalencia de tuberculosis también tienen altos niveles de infección $\mathrm{VIH}^{4}$ y resultan ser también los más pobres. En la Figura 1 se aprecia la alta concentración de casos en el continente africano.

\section{El Programa de Control de la Tuberculosis (PCT)}

La lucha antituberculosa en Chile comenzó a organizarse junto con la formación del Servicio Nacional de Salud en 1952, lo que permitió aplicar estrategias de control en todo el territorio nacional. Continuó con la aplicación de tratamientos bajo el concepto del $\mathrm{DOT}^{6}$ (sigla del inglés con que se denomina la terapia estrictamente supervisada por el personal de salud), enfoque muy diferente al habitual de entrega de fármacos a los pacientes. Con la creación posterior de una red de laboratorios de bacteriología de la tuberculosis, asesorados en su calidad por el Instituto de Salud Pública, comenzó la pesquisa bacteriológica a través de la baciloscopia de expectora- ción en sintomáticos respiratorios (tosedores con expectoración por más de 15 días) desplazando a la entonces popular radiología en formato de Abreu. En 1973 se inició la actividad de un PCT de carácter nacional, con objetivos y estrategias claramente definidas para el diagnóstico, prevención y tratamiento de la tuberculosis y con elementos de gestión y registros sistemáticos. Desde entonces el programa ha seguido mejorando, hasta que en 1997 se decidió crear un Programa Nacional de Control y Eliminación de la Tuberculosis (PROCET) con metas más definidas. La evaluación sostenida y oportuna de esta estrategia ha permitido un adecuado perfeccionamiento de las normas programáticas ${ }^{7}$.

El programa antituberculoso chileno conserva la gratuidad para todos los residentes en el país, sin limitaciones previsionales, y garantiza la pesquisa bacteriológica para la localización diagnóstica de casos pulmonares contagiosos (bacilíferos), así como el tratamiento de todas las formas de tuberculosis con esquemas normados.

\section{Situación epidemiológica de la tuberculosis en Chile}

El registro más antiguo disponible es el de la mortalidad $^{8}$. En la Figura 2 se aprecia el gran impacto que en medio siglo (1950-2000) significó la reducción de muertes por tuberculosis. Las tasas descendieron de 270 x100.000 (1903-1950) a 1,3 x 100.000 (2008).

Concomitantemente con el inicio del PCT se dispuso de registros sobre la incidencia de la tuberculosis, los que muestran una reducción de más de la mitad de las tasas de esta enfermedad cada 10 años aproximadamente. Sin embargo, a partir del año 2000 la magnitud de la declinación anual, que en la década 1990-2000 era 


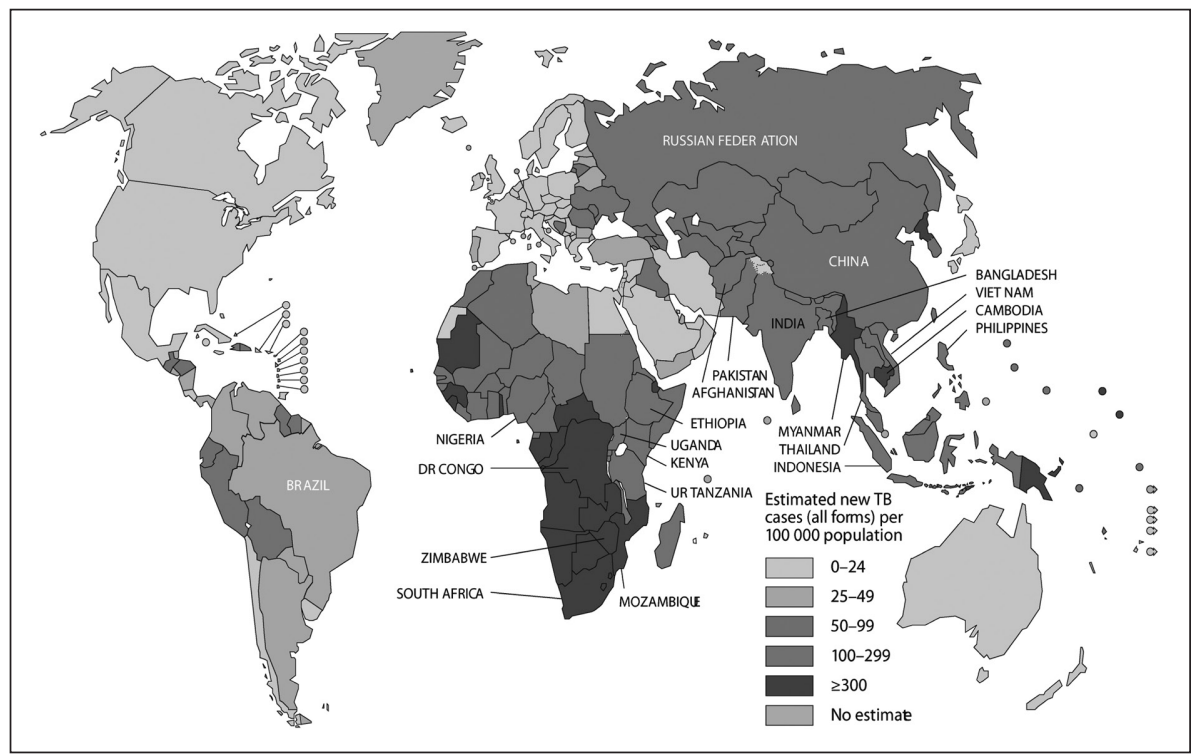

Figura 1. Incidencia estimada de tuberculosis a nivel mundial. OMS. 2010. de $-7,8 \%$, se fue reduciendo a $-4 \%$ anual en el período 2000-2008, hasta llegar a una situación estacionaria a partir del año 2009 , como muestra la Figura 3.

Además, en la Figura 4 se puede apreciar que la distribución de la incidencia en los distintos Servicios de Salud no sigue una distribución de curva normal, con 14 servicios que tienen tasas mayores que el promedio nacional. Las mayores tasas se observan en Arica e Iquique?.

\section{Grupos de riesgo de tuberculosis}

Entre las teorías acerca de este enlentecimiento en el descenso de la incidencia de la tuberculosis durante los últimos años se planteó que se debía a un aumento de las condiciones que facilitan la aparición de la enfermedad, como son los grupos de riesgo, ya sean inmigrantes, personas privadas de libertad (PPL), vagabundos (hospederías), infectados VIH y adultos mayores, los que comen-

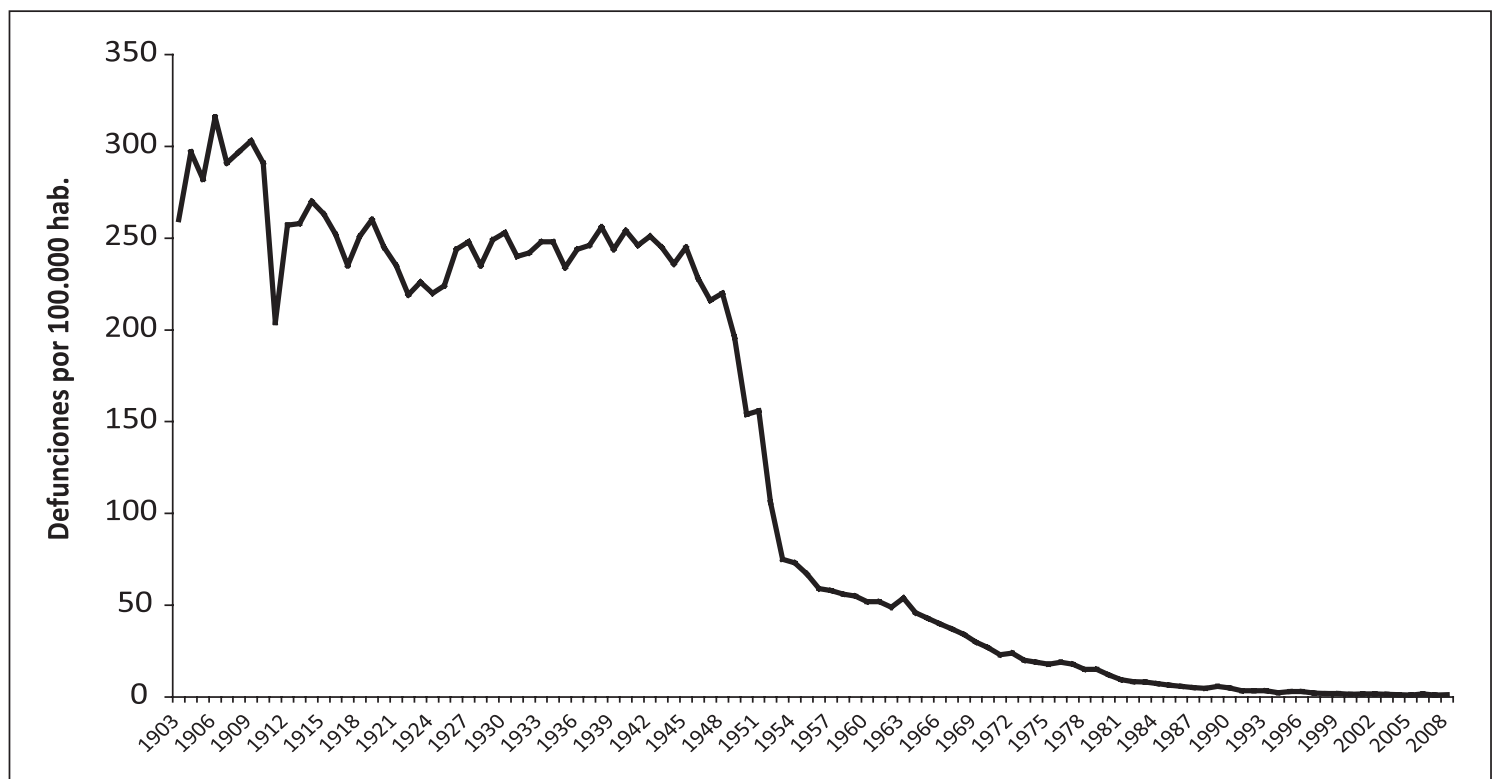

Figura 2. Tasas de mortalidad específica por tuberculosis. Chile (1903-2008). Se aprecia la disminución del riesgo de morir por tuberculosis en Chile en el período. Fuente: Programa Nacional de Control de la Tuberculosis. 
zaron a ser evaluados a partir del año 2000 en el Servicio de Salud Metropolitano Central (SSMC) ${ }^{10}$, en el que se observó que entre los años 2000-2003 un 54,4\% de los casos de tuberculosis eran atribuibles a dicha condición (Figura 5a).

La mayoría de los pacientes de Grupos de Riesgo resultaron ser adultos mayores $(33,4 \%)$, como muestra la Figura $5 b$.

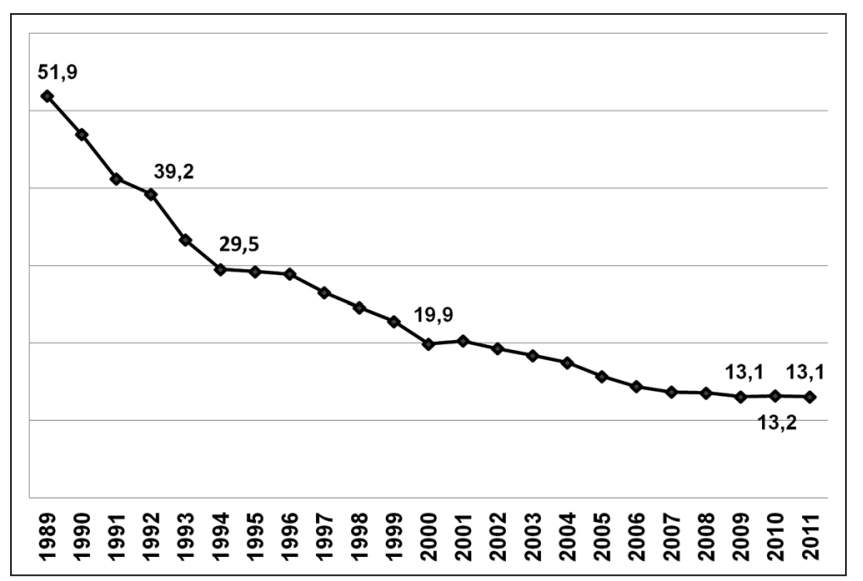

Figura 3. Tasas de tuberculosis todas las localizaciones. Chile (1989-2011).

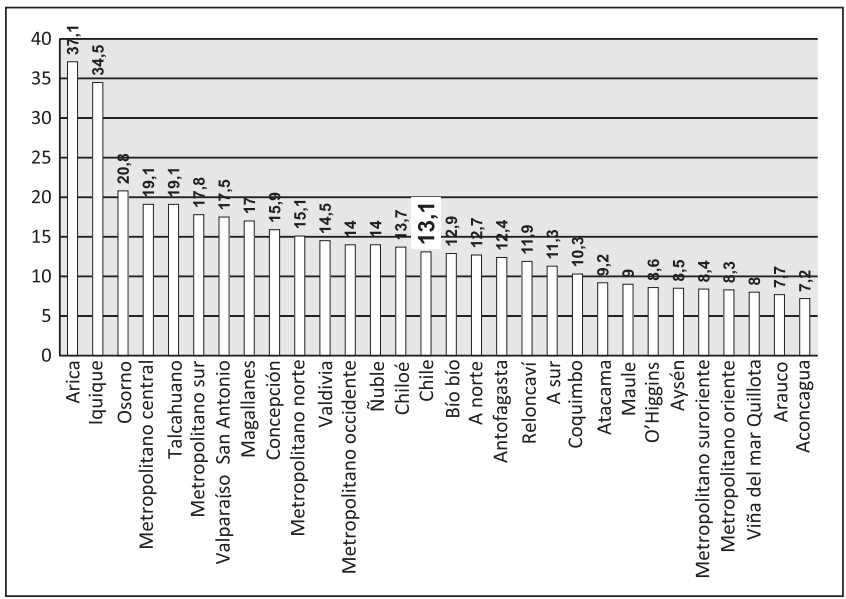

Figura 4. Incidencia de tuberculosis en todas las formas en Servicios de Salud. Chile. 2011.

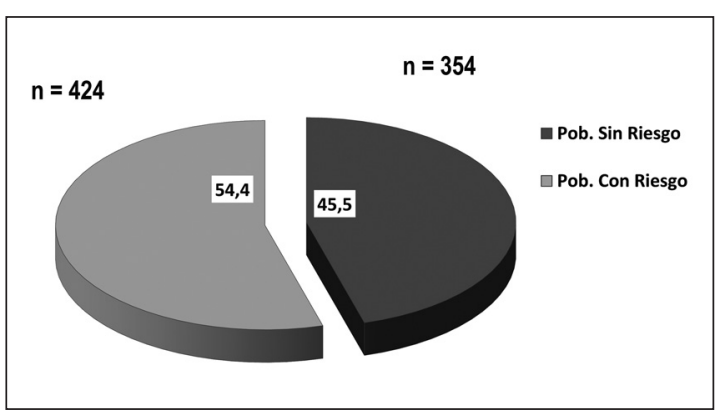

Figura 5a. Grupos de riesgo de Tuberculosis en el Servivio de Salud Metropolitano Central (SSMC), Chile: 2002-2003.
Entre los años 1998-2001 se evaluó la situación de la tuberculosis en los centros de reclusión de la Región Metropolitana, encontrando una tasa promedio de 192 x 100.000 en el año inicial de la evaluación (1998) y 222,2 x 100.000 en el año final $(2001)^{11}$. Los años siguientes arrojaron una tasa promedio de 332 x 100.000 reclusos entre 2002 y 2004 (para la población nacional de reclusos de ese período significaría cerca de 125 casos de tuberculosis en todo el país). Además, un $28 \%$ de ellos estaban asociados a infección VIH.

Entre los años 2005-2007 la Región Metropolitana evaluó su población de riesgo de tuberculosis (Figura 6), confirmando que el adulto mayor era el grupo más importante ${ }^{12}$.

El año 2009 el adulto mayor fue responsable del $19,7 \%$ de los casos de tuberculosis de la región metropolitana ${ }^{13}$, es decir, uno de cada 5 pacientes con tuberculosis era adulto mayor, siendo los otros grupos mucho menos importantes (3-4\% de todos los casos para cada uno de ellos) ${ }^{14}$ (Tabla 1).

Tabla 1. Grupos de riesgo de tuberculosis en Chile. 2009

\begin{tabular}{|lrc|}
\hline $\begin{array}{l}\text { Condición de } \\
\text { riesgo }\end{array}$ & $\begin{array}{c}\text { n de } \\
\text { casos }\end{array}$ & $\begin{array}{c}\text { Distribución } \\
\text { porcentual } \\
(\%)\end{array}$ \\
\hline Inmigrantes & 92 & 3,7 \\
Reclusos & 81 & 3,3 \\
VIH exclusivo & 93 & 3,8 \\
\hline Adulto Mayor & 487 & 19,7 \\
\hline Notificaciones & 2.475 & \\
\hline
\end{tabular}

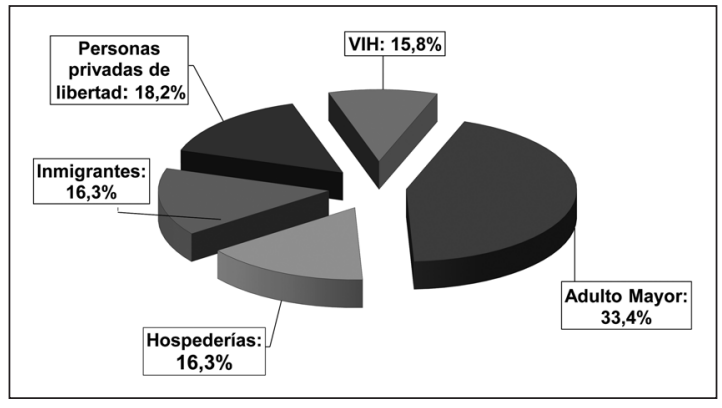

Figura 5b. Distribución Grupos de riesgo de Tuberculosis en Servicio de Salud Metropolitano Central (SSMC), Chile 2000-2003. 

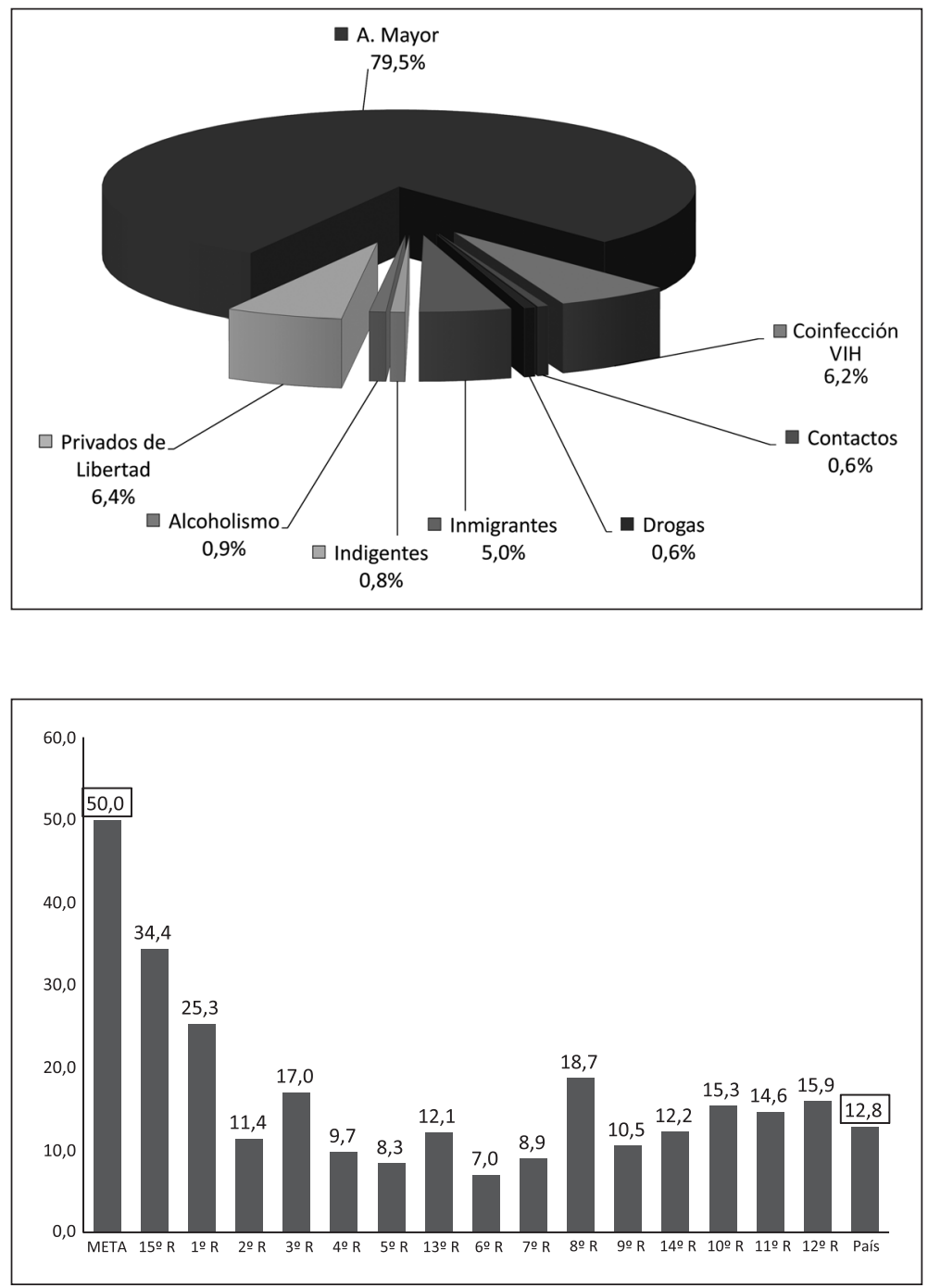

Figura 6. Distribución de Grupos de riesgo de tuberculosis en Chile. 2005 $2007(n=1.954)$.
Sin embargo, no fue posible atribuir a la presencia de estos grupos de riesgo la causa del deterioro epidemiológico actual de la tuberculosis en Chile, porque como se observa en las Figuras 5 b, 6 y 13 su magnitud no ha variado significativamente en todo este período.

\section{Localización de las fuentes de transmisión}

La mayor proporción de casos de tuberculosis pulmonar se detecta mediante la baciloscopia de expectoración. Estos casos, denominados bacilíferos, son los que mantienen la endemia en la población. Si no diagnosticamos oportunamente a estos pacientes generarán casos secundarios y empeorará la situación epidemiológica. Esta sería una de las razones principales por las cuales no ha seguido disminuyendo la incidencia de la tuberculosis en Chile.
La mayoría de los enfermos deberían ser diagnosticados en la atención primaria. Uno de los indicadores que muestra la capacidad diagnóstica es el llamado Índice de Pesquisa, que es el número de baciloscopias de expectoración realizadas por cada 1.000 consultantes adultos en la atención primaria. Con un indicador de 50 x 1.000 se puede detectar por lo menos el $70 \%$ de la incidencia. Como puede apreciarse en la Figura 7, el promedio nacional de este índice el año $2010(12,8 \times 1.000)$ está muy distante de la meta fijada por el PNT. Ese año ninguna región del país alcanzó la meta.

Al observar la cantidad de baciloscopias practicadas entre los años 2006-2010 (Figura 8), se aprecia una reducción de 193.416 exámenes. Si se calcula la suma de los déficits de diagnóstico en esos 4 años, utilizando como base la pesquisa del año 2006 y calculando la probabilidad de haber identificado como tuberculosos al $0,5 \%$ de los 


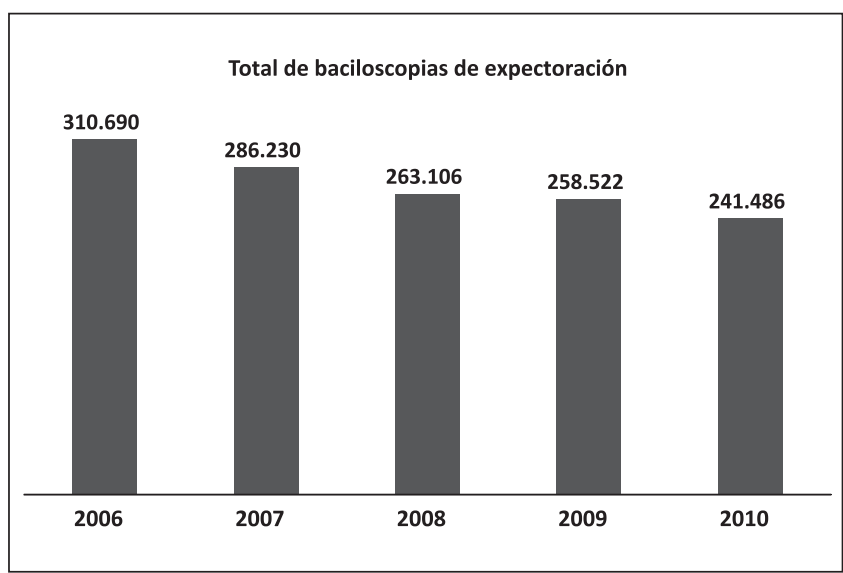

Figura 8. Baciloscopias anuales de diagnóstico. Chile 2006-2010. Capacidad de diagnóstico de tuberculosis pulmonar bacilífera según baciloscopias de expectoración realizadas durante el período.

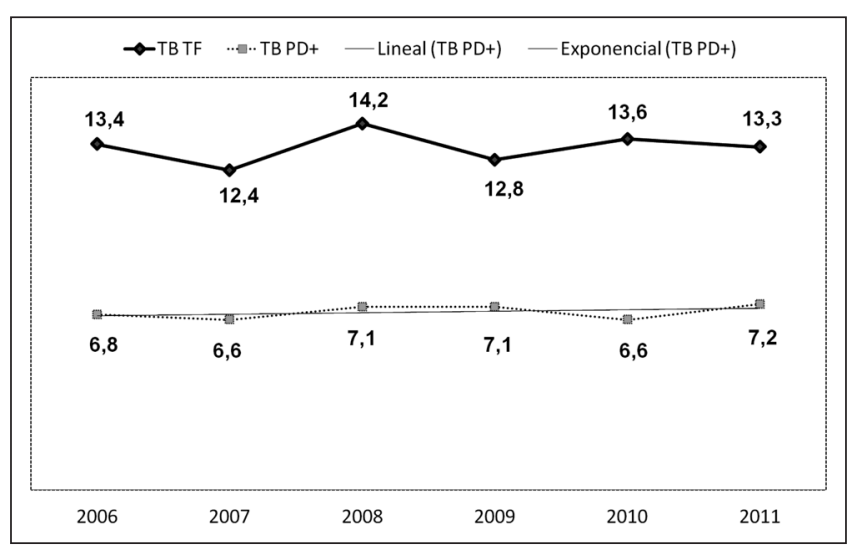

Figura 9. Incidencia de tuberculosis en todas las formas y pulmonar bacilífera. Región Metropolitana. 2006-2011. Curva superior representa la incidencia de tuberculosis en todas sus formas (TB TF: -“- ); la curva inferior representa la incidencia de tuberculosis pulmonar bacilífera (TB PD+ : -'- ).

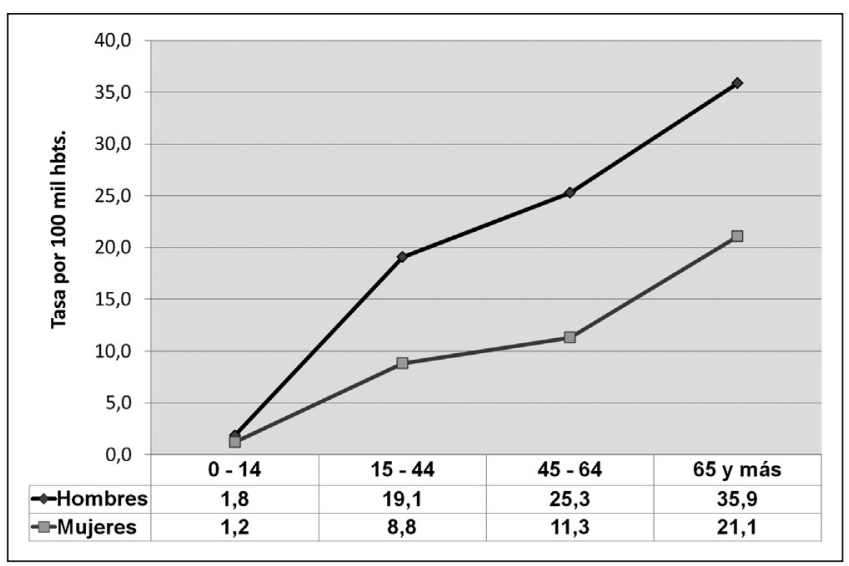

Figura 10. Incidencia de tuberculosis según género y edad en la Región Metropolitana. 2011. exámenes practicados, esto significaría que se diagnosticaron 966 casos menos, los que contribuirán a mantener la endemia tuberculosa.

\section{Situación de la tuberculosis en la Región Metropolitana}

La situación epidemiológica de la tuberculosis en la Región Metropolitana $(\mathrm{RM})^{15}$ es inquietante ya que también muestra una tendencia estable de la incidencia de la enfermedad en una población de casi 7 millones de habitantes. Aunque se mantienen las tasas, debido al aumento poblacional, el número de casos se incrementó en $11,6 \%$, al comparar los datos del año 2006 con los del 2011 (Figura 9). La tendencia es especialmente alarmante para los enfermos bacilíferos, que en el mismo lapso aumentó 5,9\%. En este período de 6 años se generaron 5.412 casos nuevos de tuberculosis, de los cuales 2.808 son pulmonares bacilíferos (52\%).

La distribución por género muestra un aumento de las tasas a mayor edad, con concentración del riesgo en hombres adultos mayores (Figura 10).

La capacidad diagnóstica de tuberculosis pulmonar, evaluada por el número de baciloscopias practicadas en la atención primaria, se encuentra seriamente comprometida, ya que existe una reducción de 98.364 exámenes de baciloscopia de pesquisa al considerar el déficit acumulativo entre el año 2006 y el 2011, lo que habría impedido el diagnóstico de 491 casos bacilíferos (Figura 11), que equivale a la cantidad de enfermos pulmonares bacilíferos de todo un año en esa región.

\section{Comunas de la Región Metropolitana prioritarias}

Actualmente, en la Región Metropolitana existen comunas con alta incidencia de tuberculosis, que mantienen tasas similares a las que tenía el país hace más de una década. Estas comunas tienen en total una población cercana a 1.140 .000 habitantes $(6,6 \%$ de la población nacional) y concentran el $18,8 \%$ de todos los casos del país. La tasa promedio de 


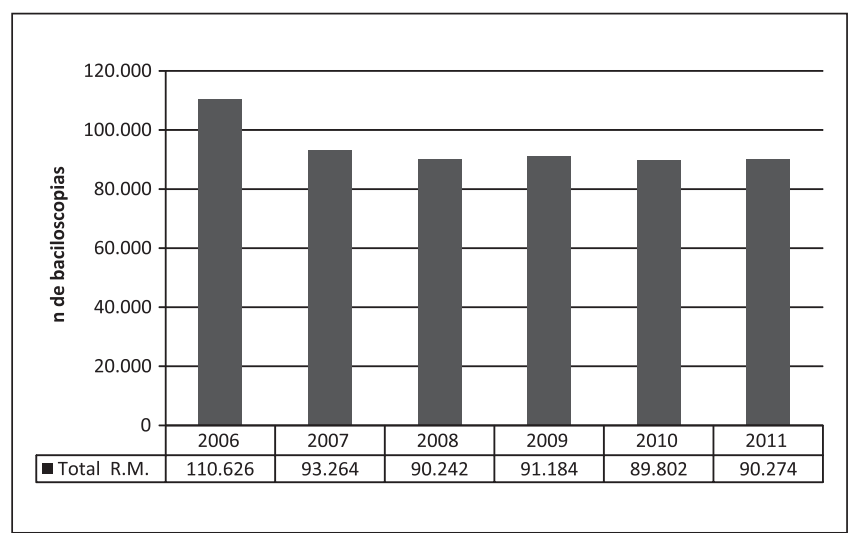

Figura 11. Número de baciloscopias anuales de pesquisa. R. Metropolitana 2006-2011. Capacidad diagnóstica de tuberculosis pulmonar bacilífera según baciloscopias de expectoración en la Región Metropolitana de Chile.

Tabla 2. Comunas de la Región Metropolitana con tasas de tuberculosis sobre 20/100.000 habitantes

\begin{tabular}{|lccc|}
\hline Comuna & Población & n casos & Tasa \\
\hline La Cisterna & 71.443 & 16 & 22,4 \\
\hline Pedro Aguirre Cerda & 92.738 & 21 & 22,6 \\
\hline Quinta Normal & 86.952 & 20 & 23,0 \\
\hline El Bosque & 168.302 & 39 & 23,2 \\
\hline Lo Espejo & 99.082 & 28 & 28,3 \\
\hline Lo Prado & 91.703 & 27 & 29,4 \\
\hline Recoleta & 124.700 & 44 & 35,3 \\
\hline San Joaquín & 77.274 & 32 & 41,4 \\
\hline Independencia & 51.277 & 24 & 46,8 \\
\hline Estación Central & 111.702 & 58 & 51,9 \\
\hline Santiago & 163.952 & 117 & 71,4 \\
\hline Total & 1.139 .125 & 426 & promedio \\
& & & 37,39 \\
\hline
\end{tabular}

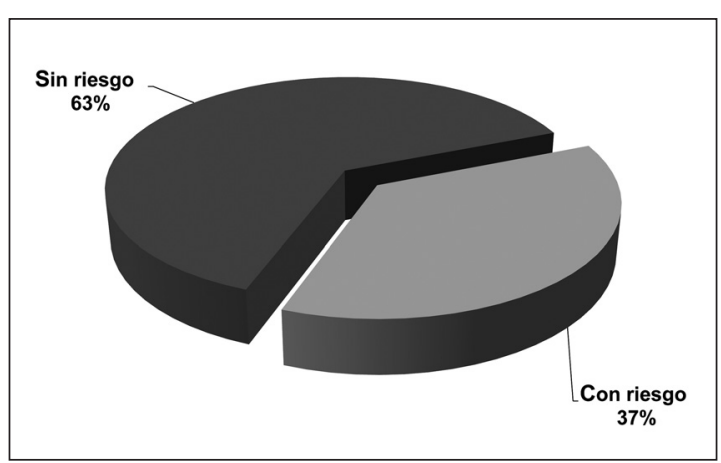

Figura 12. Proporción de grupos de riesgo de tuberculosis. Región Metropolitana. 2011. este conjunto de 11 comunas es de 37,4 $\mathrm{x} 100.000$, con un rango entre 22,4 (La Cisterna) y 71,4 (Santiago). Como tienen las mayores tasas de tuberculosis a nivel nacional, en ellas se deberán intensificar las acciones del programa (Tabla 2).

En el resto del país deberán identificarse igualmente las comunas y zonas donde se concentran las mayores tasas de incidencia de la tuberculosis, para intensificar en ellas las acciones de control.

\section{Factores de Riesgo de tuberculosis en la Región Metropolitana}

La proporción de grupos vulnerables en la Región Metropolitana es similar a la del resto del país (Figura 12). En esta región se aprecia una mayor proporción de extranjeros $(6,3 \%$ vs $3,7 \%)$, pero el resto de los otros grupos de riesgo es similar (Figura 13). Este aumento, ocasionado por extranjeros en la Región Metropolitana, no determina ninguna diferencia en la tasa regional respecto a la del resto de Chile.

Sólo el 39,3\% de la población de la Región Metropolitana se encuentra cercana a la meta de eliminación de la tuberculosis como problema de salud pública $(5 \times 100.000)$ propuesta para el año 2020, según el objetivo sanitario del Plan Nacional de Salud Pública del Ministerio $^{16}$. Su tasa actual promedio es $6,2 \times 100.000$ habitantes.

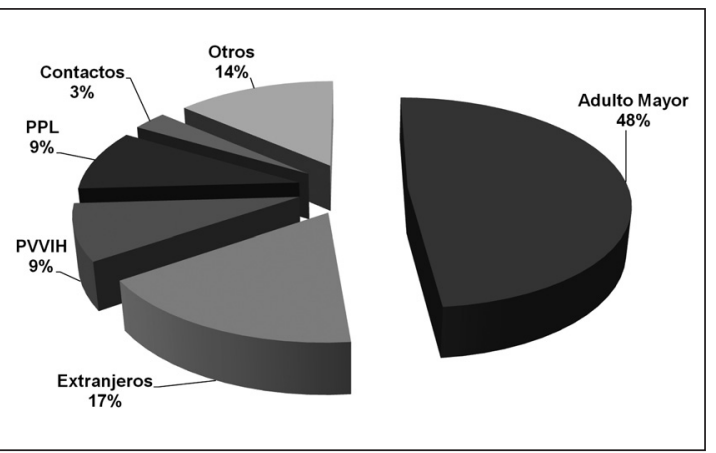

Figura 13. Distribución de los grupos de riesgo de tuberculosis. Región Metropolitana. 2011. 


\section{Conclusiones}

La tuberculosis es una enfermedad que aún causa un impacto sanitario importante en Chile y debe ser considerada como un problema de salud pública ${ }^{17}$. Es necesario intensificar el PCT reforzando sus probadas estrategias, aumentando la pesquisa de sintomáticos respiratorios consultantes en la atención primaria, servicios de urgencia y grupos de riesgo. Además debe asegurarse el estudio sistemático de los contactos de enfermos bacilíferos y el tratamiento bajo la estrategia DOTS (tratamiento de corta duración, supervisado directamente por personal entrenado $)^{18}$. Es esencial que los niveles locales reciban una permanente capacitación y supervisión desde los niveles intermedios dentro de un plan organizado y evaluado periódicamente.

Estas estrategias han conducido al país a una situación epidemiológica favorable, reconocida en toda América y el mundo, lo que nos compromete a combatir con más fuerza la enfermedad en una etapa donde se vuelve menos frecuente, los recursos para combatirla se van reduciendo, la experiencia y el conocimiento profesional de ella se va perdiendo, algunas autoridades la perciben como menos relevante en las agendas programáticas y aumenta el desconocimiento de ella en la población general. En los años posteriores al año 2000 el país muestra un descenso menor de la incidencia, la que finalmente llega a una situación estacionaria entre los años 2009-2011. Aunque se había vinculado este proceso de deterioro epidemiológico del PCT al aumento de los grupos de riesgo, en realidad el mayor problema es la pérdida de la capacidad diagnóstica de los casos pulmonares bacilíferos, reflejada en el subóptimo índice de pesquisa, lo que mantiene en circulación numerosos pacientes contagiosos no diagnosticados. A algunos especialistas les cuesta aceptar que al disminuir un problema como la tuberculosis en un país, debido a la ley de los rendimientos decrecientes, se requieren no menos, sino que más recursos para seguir avanzando en su control.

El papel de la atención primaria de salud es crucial para aumentar la cobertura y oportunidad diagnóstica de los casos pulmonares bacilíferos, ya que la demora en el diagnóstico involucra mayor riesgo comunitario por mantención de la transmisión de la infección y un peor pronóstico para los pacientes. Se debe establecer un compromiso con los directores de los consultorios para crear equipos locales de tuberculosis, asignándoles la responsabilidad de intensificar la pesquisa intra-consultorio en colaboración con otros estamentos y de asegurar un índice de pesquisa adecuado.

\section{Bibliografía}

1.- WHO. Global Tuberculosis Control. Switzerland. 2009.

2.- Control Mundial de la Tuberculosis. Informe OMS 2011.

3.- WHO. Global Tuberculosis Report. 2010.

4.- Centro de prensa de la OMS. 2005. La morbilidad y la mortalidad por tuberculosis relacionadas con el VIH alcanzan ya niveles alarmantes en África.

5.- OMS.Global Tuberculosis Report 2012.

6.- FARGA V. Hacia la erradicación de la tuberculosis. Rev Chil Enf Respir 2006; 22: 52-4.

7.- PEÑA C. Actualización del programa de control de la tuberculosis. Rev Chil Enf Respir 2008; 24: 317-22.

8.- FARGA V. Nuevos desafíos en tuberculosis. Rev Chil Enf Respir 2011; 27: 161-8.

9.- HERRERA T. PROCET Ministerio de Salud de Chile. Informe Nacional 2011.

10.- PEÑA C. Programa de control de tuberculosis Servicio de Salud Metropolitano Central. 2003.

11.- CARRILLO J. Visión de la tuberculosis en recintos penales de la Región Metropolitana. Rev Chil Enf Respir 2004; 20: 43-6.

12.- ZÚNIIGA M. PROCET Ministerio de Salud de Chile. Informe Nacional. 2007.

13.- GARCÍA C. Tuberculosis en grupos de riesgo en la Región Metropolitana 2008. Rev Chil Enf Respir 2010; 26: 105-11.

14.- GARCÍA C. PROCET Ministerio de Salud de Chile. Informe Nacional 2009.

15.- ZÚÑIGA S. SEREMI Región Metropolitana de Chile. Informe regional 2011.

16.- MINISTERIO DE SALUD DE CHILE. Los objetivos sanitarios para la década 2000-2010. Octubre 2002.

17.- ZÚÑIGA M. La eliminación de la tuberculosis como problema de salud pública. Situación de Chile en el año 2008. Rev Chil Enf Respir 2009; 25: 117-26.

18.- FARGA V, CAMINERO J A. Tuberculosis. $3^{\text {a }}$ Edición. Editorial Mediterráneo. 2011.

Correspondencia a:

Dr. Carlos Peña M.

Equipo Técnico de Tuberculosis.

Servicio de Salud Metropolitano Central.

E-mail: carpemanti@hotmail.com 